\title{
Bilateral Reversible Corneal Edema Associated With Amantadine Use
}

\author{
Salomon Esquenazi
}

\begin{abstract}
Purpose: In this article, we report a case of bilateral severe reversible corneal edema caused by amantadine therapy.

Case: A 39-year-old women was referred to us for evaluation of bilateral corneal edema. Her past medical history was significant for multiple sclerosis, anorexia, and seizures. She developed painless progressive bilateral loss of vision for the past 6 months. She was evaluated by several ophthalmologists elsewhere who felt that the patient's visual loss was secondary to a nutritional deficiency as opposed to related to multiple sclerosis. She was started on vitamin B-12 medication without improvement in her symptoms. She was then evaluated by neuro-ophthalmology. The examination revealed severe bilateral corneal edema and was referred to our corneal service for further evaluation of her corneal condition. Our examination revealed best corrected visual acuity of $20 / 400$ bilaterally. Corneal thickness was $940 \mu \mathrm{m}$ in the right eye and $802 \mu \mathrm{m}$ in the left. Color vision was intact. Conjunctivas were white bilaterally. Cornea evaluation revealed diffuse stromal edema and Descemet's folds and microcystic subepithelial edema with to guttae noted. Anterior chambers were deep and quiet. A specular microscopy revealed significant pleomorphism and polymegathism with an endothelial cell count of 1,504 cells in the right eye and 1,596 in the left eye.

Results: Review of the patient's medical information revealed therapy with amantadine 2 months prior to the appearance of the patient's symptoms as a means to control the patient's tremors. The patient experienced rapid resolution of the corneal edema within the next 2 months after discontinuation of the agent with recovery of best corrected visual acuity of 20/40 in the right eye and 20/30 in the left.

Conclusions: In cases of unexplained corneal edema and in the absence of any identifiable ocular cause, a review of toxic effects of systemic medications should be performed. Early diagnosis may prevent irreversible endothelial damage. Amantadine can cause endothelial failure and needs to be considered as part of the differential diagnosis of corneal edema.
\end{abstract}

\section{Introduction}

$\mathrm{A}$ MANTADINE, WHICH WAS ORIGINALLY used as an antiviral agent, is currently prescribed in reducing tremors of Parkinson's disease and off-label to treat fatigue in multiple sclerosis. The mechanism of action is not fully understood. ${ }^{1}$

Very few reports have been published regarding ocular complications of amantadine use. The ocular side effects reported include visual loss, mydriasis, punctuate subepithelial opacities, and stromal and epithelial edema and oculogyric crisis. ${ }^{2,3}$ Few reports have been published regarding ocular complications of amantadine use. Recently 3 articles on corneal edema associated with amantadine use were published. ${ }^{4-6}$
The present report describes the clinical features of one patient with amantadine-associated corneal edema that resolved completely after cessation of the agent. The review was conducted with a waiver from the institutional review board of the Rand Eye Institute and conforms to health insurance portability and accountability act (HIPPA) regulations.

\section{Case Report}

A 39-year-old woman was referred to our corneal service for evaluation of bilateral corneal edema. Her past medical history was significant for skull fractures that she had after head traumas that she had 11 and 9 years ago, seizure

Ocular Surface Center at Rand Eye Institute, Deerfield Beach, Florida, and Department of Ophthalmology, LSU Eye and Neuroscience Center, Louisiana State University Health Sciences Center, New Orleans, Louisiana. 
disorder that started after her second skull fracture treated with topiramide and relapsing-remitting multiple sclerosis without optic neuritis diagnosed 9 years ago with three active plaques noted on the last magnetic resonance imaging (MRI) done in November of 2008. Additionally, she underwent a left internal cerebral artery aneurysm clipping 2 years ago and anorexia nervosa diagnosed 3 years ago. She developed painless progressive bilateral loss of vision for the past 6 months. She was evaluated by several ophthalmologists elsewhere who felt that the patient's visual loss was secondary to a nutritional deficiency as opposed to related to multiple sclerosis. She was started on vitamin B-12 medication without improvement in her symptoms. She was then evaluated by neuro-ophthalmology. The examination revealed severe bilateral corneal edema. It was felt that the decreased visual acuity was consistent with a bilateral corneal edema noted in that visit as opposed to an underlying optic neuropathy given the fact that her color vision was intact bilaterally in spite of her severely depressed central acuity and intact fields on confrontation and diffuse depression with automated perimetry. She was then referred to our corneal service for further evaluation of her corneal condition.

Our examination revealed best corrected visual acuity of 20/400 bilaterally. Intraocular pressures were $13 \mathrm{mmHg}$ in the right eye and $12 \mathrm{mmHg}$ in the left eye. Corneal thickness was $940 \mu \mathrm{m}$ in the right eye and $802 \mu \mathrm{m}$ in the left. Color vision was intact. Conjunctivas were white bilaterally. Cornea evaluation revealed diffuse stromal edema and Descemet's folds and microcystic subepithelial edema with to guttae noted (Fig. 1). Anterior chambers were deep and quiet. Angles were wide open. The lenses revealed trace nuclear sclerotic changes. Retinal examination revealed healthy optic nerve head with sharp edges and pink rims with physiologic cupping. Macula was healthy and the peripheral retina looked normal. A specular microscopy revealed significant pleomorphism and polymegathism with an endothelial cell count of 1,504 cells in the right eye and 1,596 in the left eye.

When interrogated regarding medications used, the patient stated that she was currently taking fluoxetine 20 mg/day, amantadine $100 \mathrm{mg} /$ day, bupropion $100 \mathrm{mg} /$ day, cepaxone $20 \mathrm{mg}$ subcutaneously/day, topiramate $50 \mathrm{mg}$ twice a day, and clonazepam $2 \mathrm{mg} /$ day. Amantadine was started 8 months before she sought treatment at a dose of $2,100 \mathrm{mg}$ twice daily as a means to control the patient's tremors and fatigue. Prior consultation with the patient's neurologist, the agent discontinued and the patient was started on topical prednisolone acetate $1 \%$ eyedrops 4 times a day. The patient experienced rapid resolution of the corneal edema within the next 2 months with recovery of best corrected visual acuity of 20/40 in the right eye and 20/30 in the left. The steroid medication was rapidly tapered off within the first 2 months of treatment. Repeated pachymetry measurements showed a corneal thickness of $562 \mu \mathrm{m}$ in the right eye and $553 \mu \mathrm{m}$ in the left eye. Repeated specular microscopy revealed normal endothelial morphology with similar cell counts as in the previous examination. The patient subsequently returned to her referring ophthalmologist for continued follow-up care.

\section{Discussion}

The author reviewed all the neurological medications implicated in corneal toxicity. ${ }^{7}$ Although many of those have been associated with ocular complications, only 2 are reported to be associated with corneal toxicity: amantadine ${ }^{2,3}$ and dopamine ${ }^{8}$ (levodopa-carbidopa). Of the 2 medications listed amantadine has been more frequently associated to corneal toxicity than dopamine.

Amantadine hydrochloride was developed as prophylaxis against influenza A in 1959. Subsequently, it was found to be effective against Parkinson's disease and has been used to treat tardive dyskinesia associated with antipsychotic medications and asthenopia associated with multiple sclerosis.

The pathogenesis of endothelial failure after amantadine use is poorly understood. Recent histopathologic analysis show severe corneal endothelial cell damage. ${ }^{3}$ Several areas of denuded endothelial cells were noted under scanning electron microscopy. No inflammatory cells have been noted in the pathology specimens confirming that no inflammatory injury is produced. Waring ${ }^{9}$ in 1982 stated that the medication may cause stress on the endothelial cells and described the presence of a posterior collagenous layer by transmission electron microscopy.

Our patient was also using topiramide, which has been reported to produce corneal thickening. ${ }^{10}$ The mechanism of central corneal thickening may be associated with the carbonic anhydrase inhibitor activity of topiramate.
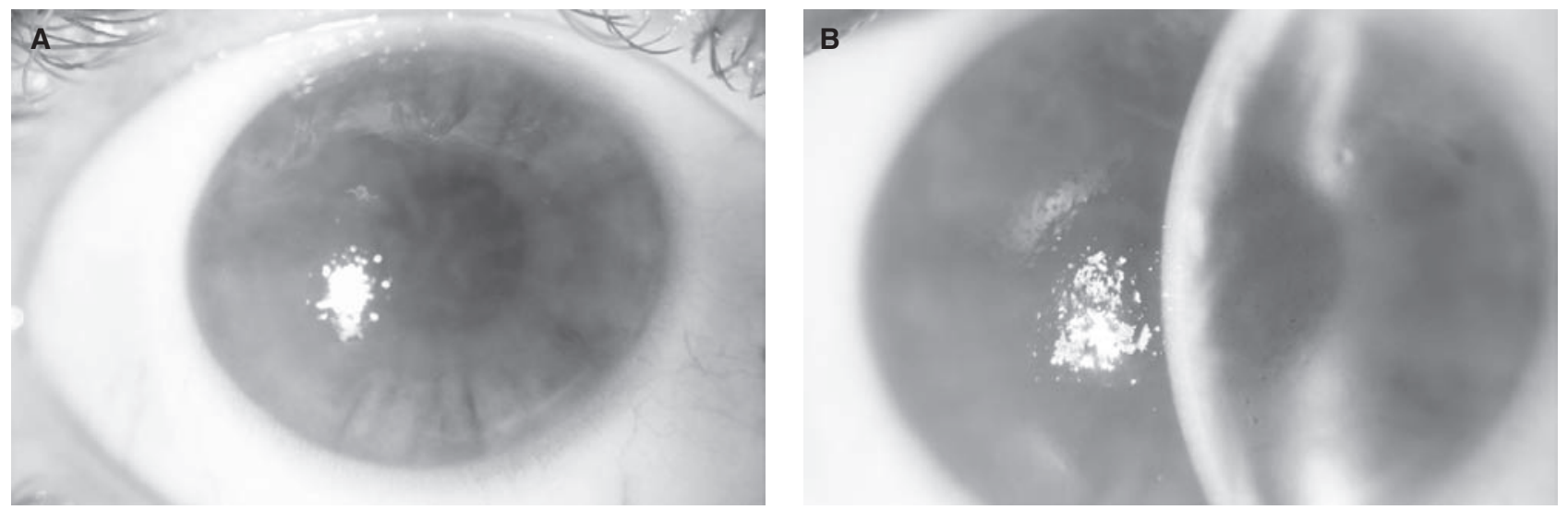

FIG. 1. Slit lamp appearance of the (A) right and (B) left eyes revealing diffuse stromal edema, Descemet's folds, and microcystic subepithelial edema on the initial evaluation. 
Carbonic anhydrase isoenzymes have been shown in the corneal endothelium as well as in the pigmented and nonpigmented epithelium of the ciliary body in patients taking topiramide. Additionally, the patient was using fluoxetine that has been implicated in inhibiting potassium transport pathways underlying volume regulation in cultured bovine corneal endothelial cells. ${ }^{11}$ To the best of my knowledge, no reports of corneal edema related to any of these medications have been published in the medical literature. However, its concomitant use with amantadine may have a contributory effect on the significant corneal edema seen clinically.

A recent review of a large national database determined that $0.27 \%$ of patients who were dispensed amantadine were diagnosed with corneal edema or Fuchs dystrophy during the 2-year study period..$^{12}$ Given the high number of patients who are taking amantadine systemically who have not developed corneal toxicity, we believe that certain eyes are, for yet unknown reason, susceptible to endothelial damage from amantadine. This may represent an idiosyncratic hypersensitivity to the drug. Alternatively, the amantadine toxicity may be dose-dependent with some patients having the ability to have higher concentrations in the aqueous humor than others, and thereby suffering endothelial damage sooner than others.

Most reports demonstrate significant edema soon after institution of amantadine therapy with resolution of the edema after discontinuation of the agent. ${ }^{2,3,10}$ However on report demonstrated chronic irreversible corneal edema un-responsive to amantadine discontinuation that required corneal transplantation. ${ }^{3}$ This finding may support the hypothesis of a dose-dependent toxicity and the fact that delaying the diagnosis could lead to irreversible endothelial failure. At the present time, however, the pathogenesis of endothelial toxicity with amantadine use is still unknown and it may even be multifactorial in origin.

\section{Conclusions}

Amantadine can cause corneal edema that usually begins several months after institution of therapy. It is usually reversible after cessation of the agent. In cases of corneal edema in the absence of any identifiable ocular cause, a review of the toxic effects of systemic medications should be undertaken. Amantadine must be considered always as a possible cause in the differential diagnosis of toxic endothelial dysfunction.

\section{Acknowledgment}

This work was supported by P20RR021970 (LSU Translational COBRE Grant) from the National Institute of Health.

\section{Author Disclosure Statement}

The author has no financial interest in any product mentioned in this manuscript.

\section{References}

1. Boerio, D., Lefaucher, J.P., Hogrel, J.Y., et al. Pathophysiology and treatment of fatigue in Multiple Sclerosis. Rev. Neurol. 162:311-320, 2006.

2. Hughes, B., Flynn, S.B., and Brodsky, M.C. Reversible amantadine-induced corneal edema in an adolescent. Cornea. 23:823-824, 2004.

3. Jeng, B.H., Galor, A., Lee, M.S., et al. Amantadine associated corneal edema potentially irreversible even after cessation of the medication. Ophthalmology. 115:1540-1544, 2008.

4. Naumann, G.O., and Schlotzer-Schrehardt, U. Amantadineassociated corneal edema. Ophthalmology. 116:1230-1231, 2009.

5. Pond, A., Lee, M.S., Hardten, D.R., et al. Toxic corneal oedema associated with amantadine use. Br. J. Ophthalmol. 93:281, 2009.

6. Dubow, J.S., Rezak, M., and Berman, A.A. Reversible corneal edema associated with amantadine use: an unrecognized problem. Mov. Disord. 23:2096-2097, 2008.

7. Hadjikoutis, S., Morgan, J.E., Wild, J.M., et al. Ocular complications of neurological therapy. Eur. J. Neurol. 12:499-507, 2005.

8. Nakamagoe, K., Ohkoshi, N., Fugita, T., et al. Keratitis and corneal edema associated with levodopa use- a case report. Rinsho. Shinkeigaku. 36:886-888, 1996.

9. Waring, G.O. III. Posterior collagenous layer of the cornea: ultrastructural classification of abnormal collagenous tissue posterior to Descemet's membrane in 30 cases. Arch Ophthalmol. 100:122-134, 1982.

10. Kerimoglu, H., Tokgoz, M., Ozturk, B., et al. Topiramate induced acute-onset myopia and central corneal thickening: Pentacam Scheimpflug imaging findings. Can. J. Ophthalmol. 44:222-223, 2009.

11. Hara, E., Reinach, P.S., Wen, Q., et al. Fluoxetine inhibits K transport pathways underlying volume regulation in corneal endothelial cells. J. Membr. Biol. 171:75-85, 1999.

12. Chang, K.C., Kim, M.K., Wee, W.R., et al. Corneal endothelial dysfunction associated with amantadine toxicity. Cornea. 27:1182-1185, 2008.

Received: March 28, 2009

Accepted: August 28, 2009

Address correspondence to: Dr. Salomon Esquenazi Department of Ophthalmology LSU Eye and Neuroscience Center 2020 Gravier Street Suite D, 8th Floor New Orleans, LA 70112

E-mail: sesque@lsuhsc.edu 
